\title{
Dynamic Light Scattering Instrument with a Time Interval Digitizer
}

\author{
Norio Nemoto, Yoshisuke Tsunashima, and \\ Michio KURATA \\ Institute for Chemical Research, Kyoto University, \\ Uji, Kyoto 611, Japan.
}

(Received September 5, 1980)

\begin{abstract}
A computer-based dynamic light scattering instrument with a time interval digitizer has been constructed for the study of the dynamics of polymers in solution. The photon counting scheme is basically the same as that developed by Matsumoto et al. [Rev. Sci. Instrum., 47, 861 (1976)] but has been refined by the incorporation of a new interface with DMA to reduce the dead time to $310 \mathrm{~ns}$. The total number of channels is as many as 512 . Our instrument allows for an accurate measurement of the intensity autocorrelation function for very weak scatterers at six discrete scattering angles from $10^{\circ}$ to $150^{\circ}$, and makes possible determination of the translational diffusion coefficients of polystyrene-latex particles in water within an accuracy of one percent. Its capability is also demonstrated in measurements on dilute benzene solutions of narrow-distribution polystyrene as well as aqueous suspensions of mixtures of PS-latex particles.
\end{abstract}

KEY WORDS Dynamic Light Scattering / Time Interval Digitizer / DMA / Mini Computer / Translational Diffusion Coefficient / Autocorrelation Function / Polystyrene / Polystyrene Latex / Decay Rate / Scattering Vector/

Since Pecora's pioneering work, ${ }^{1,2}$ dynamic light scattering (DLS) has proved to be a powerful tool for studying the dynamics of polymer solutions over a wide concentration range. DLS has also found broader applicability in the study of simple liquids near the critical point, binary mixtures, liquid crystals, and polymeric gels. ${ }^{3,4}$

Recently, Matsumoto et al. ${ }^{5}$ designed a computer-controlled software correlator and showed that it could determine the translational diffusion coefficient $D$ of polystyrene latex within an uncertainty of $5 \%$. This accuracy is not higher than the one achieved by ordinary hardware correlators, but the flexibility of the software correlator system offers a greater potential for future application, as was noted by $\mathrm{Chu}^{6}$

In the study of polymer dynamics in solutions in which we are interested, the intramolecular or gel mode often overlaps the translational diffusive mode, and the separation of the two modes requires a very accurate determination of the autocorrelation function $A(\tau)$.

We constructed a dynamic light scattering system with a software correlator, essentially based on the design reported by Matsumoto. However, the following modifications have been introduced to improve measurement accuracy: (1) incorporation of DMA to reduce the dead time from $800 \mathrm{~ns}$ to $310 \mathrm{~ns}$; (2) increase of the total number of channels to 512 ; (3) an improved procedure to obtain the true statistical average for photon correlation; and (4) careful optical alignments. We believe that these modifications bring about a reliability equal to or greater than that of available hardware correlators. Measurements on polystyrene latex spheres, their mixtures, and polystyrene in benzene by the present apparatus show that our expectations have generally been realized.

\section{INSTRUMENTATION}

The block diagram of our dynamic light scattering instrument is shown in Figure 1. The light source was the $488 \mathrm{~nm}$ line of an argon ion laser (Coherent CR-6) with an oven stabilized etalon which provided completely monochromatic light. 


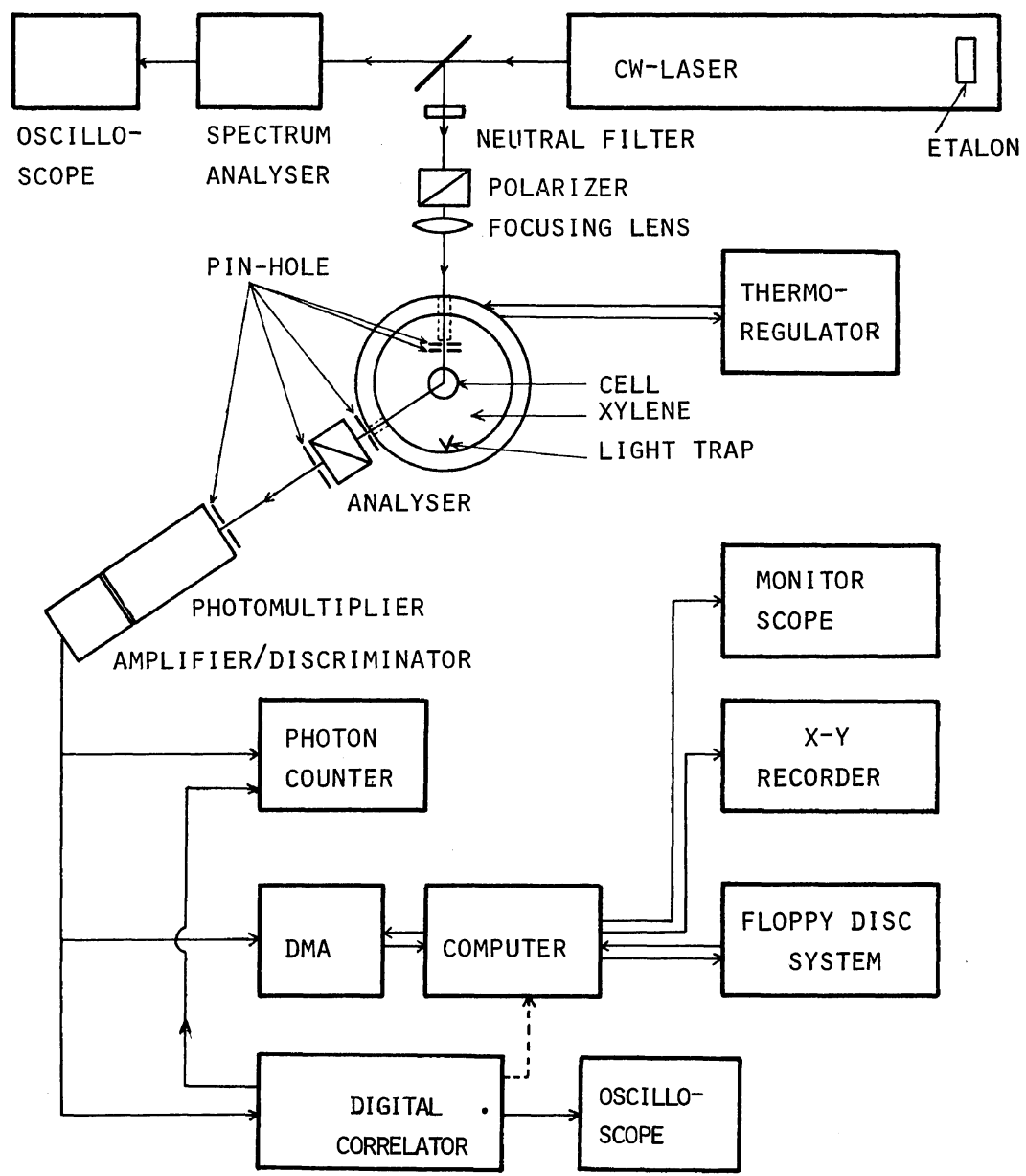

Figure 1. Block diagram of the dynamic light scattering instrument.

The output of the laser was monitored by a spectrum analyzer (Tropel model 240) to ensure that the laser was single moded before and after measurements. The incident beam was passed through a disk-shaped rotatable neutral density filter to reduce the power of the single moded light, and also through a modified Glan-Foucault prism (an extinction ratio $\leq 1 \times 10^{-5}$ ) to assure vertical polarization, and then focused by a lens on the center of a cylindrical sample cell. NMR tubes (O.D. $=12 \mathrm{~mm}$ ) without any flaws were used as sample cells. The cell was immersed in dust-free xylene (Nakarai Co., reagent grade), and the temperature was controlled within $\pm 0.02^{\circ} \mathrm{C}$ by circulating aqueous alcohol through an outer bath and an external thermostating bath.
Detection of the light scattered from the solution was made at six fixed positions corresponding to scattering angles of $10^{\circ}, 30^{\circ}, 60^{\circ}, 90^{\circ}, 120^{\circ}$, and $150^{\circ}$. The scattered light was vertically polarized by a modified Glan-Foucault prism (an extinction ratio $\leq 1 \times 10^{-5}$ ), passed through two apertures $30 \mathrm{~cm}$ apart from each other, and projected onto the photocathode of a photomultiplier (Hamamatsu TV model 464). The analyzer, the two aperture housings and the removable photomultiplier were mounted on an optical bench at the site of each angle and protected from stray light by a light shield. Six optical benches were fixed on a semicircular aluminum plate with a radius of $69.95 \mathrm{~cm}$ in such a way that the directions of their center lines coincided with those of corresponding scattering 


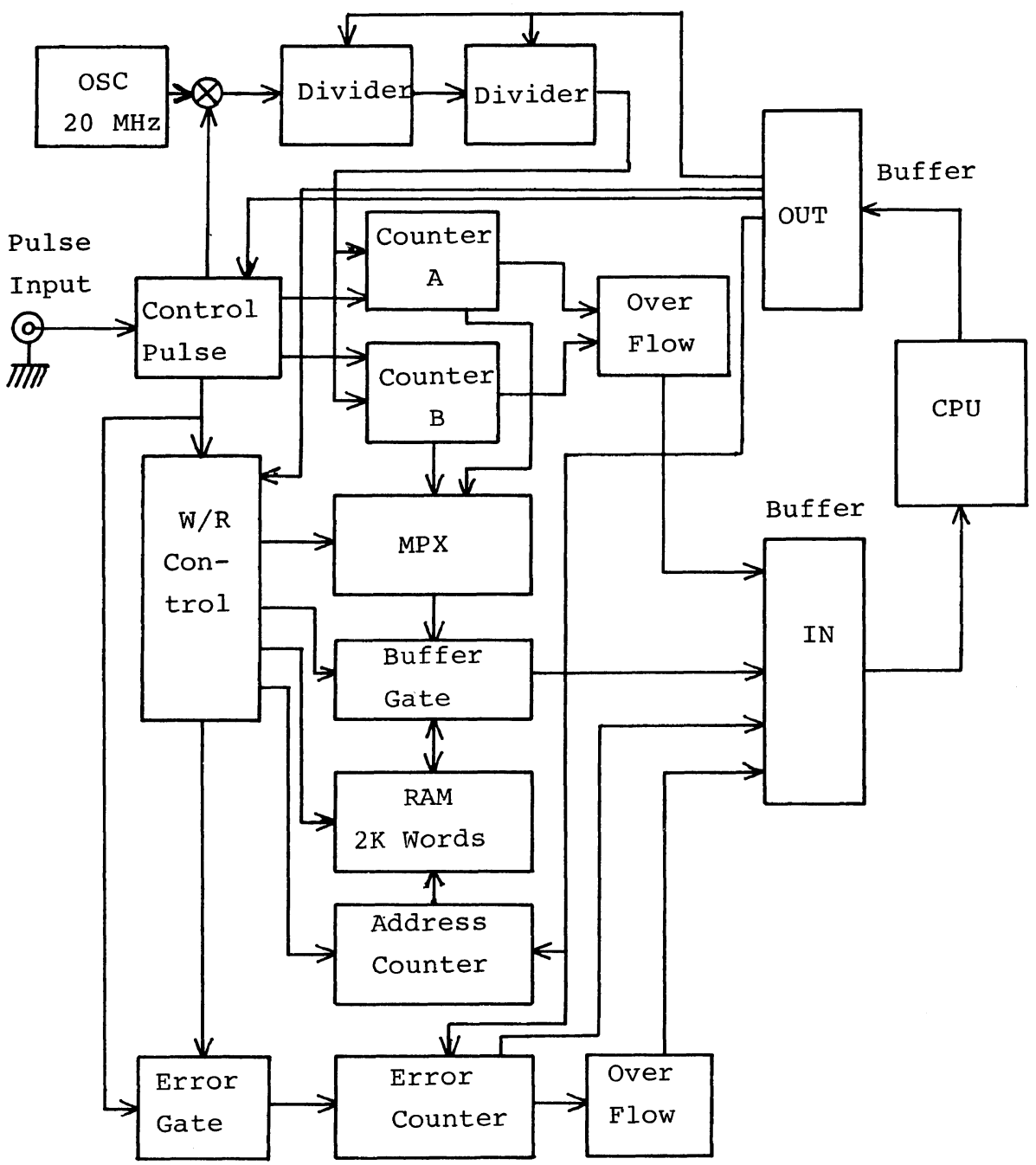

Figure 2. Block diagram of time interval digitizer. Two multiplexers connected to the buffer OUT are omitted from this figure for convenience.

angles calculated geometrically. This arrangement was suitable for simultaneous measurements at more than one angle. The whole assembly was mounted on a rigid table supported by four isolation air servo mounts (SWCC, type O) with a resonant frequency of about $1 \mathrm{~Hz}$.

The output signal from the photomultiplier was linearly amplified and discriminated through a discriminator (Hamamatsu TV, C 1050), which produced positive logic pulses with an $80 \mathrm{~ns}$ width. The pulses were fed into either a photon counter (Ortec, model 9315) for intensity measurements or a DMA interface for evaluation of the normalized autocor- relation function $A(\tau)$. The interface was connected to a mini computer operated by a floppy disk system (DEC, PDP-11 V03).

Figure 2 shows the block diagram of the interface. Since the basis of the photon counting method with a time interval digitizer (TI method) has already been described in ref 5 , here we simply list our modifications. First, a $20 \mathrm{MHz}$ crystal oscillator and a pair of dividers shown on the top of Figure 2 generated clock pulses whose frequency could cover a fairly wide range from $20 \mathrm{MHz}$ to $250 \mathrm{~Hz}$ and could be varied on a fine scale as $1 / 2,1 / 3,1 / 4,1 / 5$, $1 / 6$, and $1 / 8$. Second, a $2 \mathrm{~K}$ words RAM (DMA) and 
an address counter were connected to the digitizer. Digitized data of time intervals between neighbouring pulses in an input pulse train were stored first in the RAM by addressing from 1 to 2048 and then transferred to the computer for counting the number $X(i)$ of all possible pairs of pulses whose time intervals were $i$ and then storing this data in the $i$-th channel $(i=0-511)$. The adaptation of the DMA reduced the dead time to as little as $310 \mathrm{~ns}$, thus enabling accurate measurements of decay rates $\Gamma_{\mathrm{q}}$ at scattering angles up to $150^{\circ}$.

The function $A(\tau)$ was also calculated in a slightly different manner. Matsumoto et al. calculated $A(\tau)$ by using $X(i)$ obtained from a limited number of pulses, say 8,000 and improved $S / N$ ratios by taking the average of repeated measurements. However, the number of data, 8,000 , is insufficient for evaluating the statistical average appearing in the definition of $A(\tau)$. This averaging also should be made for data obtained within a constant period of time. To overcome this shortcoming measurements were repeated $N$ times until the accumulated number of $X(1)$ reached a value slightly less than $2^{15}$, the maximum non-negative value allowed for the 16-bit memory of the PDP-11. Usually, the value for $N$ was taken between 150 and 300. $A(\tau)$ was then obtained from eq 1 ,

$$
A(\tau)=\frac{\langle X(i)\rangle}{(2048 /\langle X(512)\rangle)^{2}(\langle X(512)\rangle-i)}
$$

Here $\langle X(i)\rangle$ represents $X(i) / N, X(512)$ is the sum of time interval data from 1 to 2048 . $A(0)$ was set equal to two. The second term in the denominator of eq 1 represents the correction that, for high $i$, the time interval between the $j$-th pulse and the last pulse becomes less than $i$ with increasing $j$, resulting in the reduction of $X(i)$.

For the simplest case in which $A(\tau)$ can be expressed by a decay curve of single exponential type as,

$$
A(\tau)=1+\beta \mathrm{e}^{-2 \Gamma} q \tau \mid+\delta
$$

where,

$$
\Gamma_{\mathrm{q}}=D q^{2}
$$

$\Gamma_{\mathrm{q}}$ or $D$ was estimated by using the PDP-11 computer. In eq $2, \beta$ is the amplitude and $\delta$ is the quantity which expresses a very small deviation of the base line $\left(=\lim _{\tau \rightarrow \infty} A(\tau)\right)$ from unity due to either the drift of the laser power or to the presence of large dust particles. $q$ is the scattering vector $\left(=\left(4 \pi n / \lambda_{0}\right) \sin (\theta / 2)\right)$, with $n$ the refractive index of the solvent and $\lambda_{0}$ the wavelength of the incident beam in vacuum. When a more detailed analysis by the method of a higher order cumulant ${ }^{7}$ or the histogram method ${ }^{8}$ was required, the FACOM M160AD computer at this insitute was used.

The output signal from the discriminator also can be fed into the Malvern 72-ch. digital correlator to obtain $A(\tau)$ (SR method) after conversion to negative pulses.

The uncertainty in $q$ due to finite acceptance angles formed by the two apertures, geometrical arrangements of the detection optics, and the divergence of the incident beam was made less than $0.5 \%$ for any scattering angles from $30^{\circ}$ to $150^{\circ}$. However, it amounted to $1.2 \%$ for $\theta=10^{\circ}$, since we were forced at this angle to use the apertures with diameters of 0.1 and $0.3 \mathrm{~mm}$ to obtain reliable $A(\tau)$ data.

Here, we remark on the differences between the TI and SR methods. We found that amplitudes $\beta$ of $A(\tau)$ obtained by the TI method were usually about one and half as large as those by the SR method for the same samples, and also that the former method allowed us to obtain data of the same quality by use of scattered intensity several times lower than that required by the latter. This indicates that the TI method is good for materials whose scattering is very weak. On the other hand, the TI method utilizes all input pulses in the calculation of $A(\tau)$ irrespective of their nature. For a precise determination of $\Gamma_{\mathrm{q}}$, therefore, unnecessary pulses originating from reflected and stray light, shot noise, and dark current, should be minimized by any means. The conventional use of three apertures close to the sample cell, as shown in Figure 1, was sufficient to cut off reflected rays. Stray light was reduced to a negligible level by taking the distance between the cell and the photocathode as large as $45 \mathrm{~cm}$. The contribution from the shot noise was excluded by an appropriate electronic device. The isolation of the instrument from external disturbing forces must be also complete, since quite small oscillations of xylene in the bath changed $A(\tau)$ from a single exponential type to a periodically decaying curve for the TI method. However, this effect was not appreciable in the SR method. Finally, the selection of photomultipliers which produce mini- 
mum after-pulse was relatively important for estimating decay rates faster than $100,000 \mathrm{~s}^{-1}$.

\section{PERFORMANCE OF THE INSTRUMENT}

Measurements on Suspensions of Polystyrene-Latex

\section{Particles}

Two polystyrene-latices of almost uniform size (Dow Chemical, diameters, $d$, equal to 0.176 and $0.091 \mu \mathrm{m}$ ) were used as samples for the calibration of the instrument. Original suspensions in water $(10 \mathrm{wt} \%)$ were sonicated for $15 \mathrm{~min}$ at room temperature and diluted by distilled water which had been filtered through a Milipore filter of 250A nominal pore size. The diluted suspensions were filtered again through a Milipore filter of $0.22 \mu \mathrm{m}$ nominal pore size before use. Intensity autocorrelation function measurements with the time interval digitizer (TI method) were made at six different scattering angles on one sample $(d=0.091 \mu \mathrm{m})$, and at five angles excepting $10^{\circ}$ on the other sample $(d=0.176 \mu \mathrm{m}) . A(\tau)$ were also measured by the digital correlator (SR method) to check the agreement of results by these two different methods. Figure 3 shows the raw data obtained by the TI method for a dilute aqueous suspension of the PS-latex $\left(c=5.5 \times 10^{-4} \mathrm{wt}^{\circ} / d=0.176 \mu \mathrm{m}\right)$ at $\theta=30^{\circ}$. Experimental conditions were $\lambda=488 \mathrm{~nm}$,
$T=25^{\circ} \mathrm{C}$, the clock pulse interval $\left(T_{\mathrm{c}}\right) 4 \mu \mathrm{s}$, the total accumulation' number 2100 , and the mean time interval between successive pulses, $150 \mu \mathrm{s}$.

The solid curve in Figure 3 represents the values of $A(i)$ calculated from eq 2 by determining the three parameters, $\beta, \Gamma_{\mathrm{q}}$ and $\delta$ by the least-squares method (the normal eq. fit). The curve fits very well the experimental points, and the value of $816 \pm 2 \mathrm{~s}^{-1}$ was obtained for $\Gamma_{\mathrm{q}}$. The curve-fitting was also made using the cumulant expansion truncated at the 2nd order (the 2 nd order fit). In this analysis, the base line is fixed to unity, and the first and second cumulants, $K_{1}\left(\equiv\left\langle\Gamma_{\mathrm{q}}\right\rangle \equiv\langle D\rangle_{\mathrm{z}} q^{2}\right)$ and $K_{2}(\equiv\langle\Gamma-$ $\left.\left.\left\langle\Gamma_{\mathrm{q}}\right\rangle\right\rangle^{2}\right)$ can be evaluated. This fitting gave $818 \pm$ $2 \mathrm{~s}^{-1}$ for $\Gamma_{\mathrm{q}}$. It is remarkable that the two values, 816 and 818 , agree to an accuracy of $0.5 \%$ with 819.5 , the value of $\Gamma_{\mathrm{q}, \text { calc }}$ given by the EinsteinStokes relationship:

$$
\Gamma_{\mathrm{q}, \mathrm{calc}}=k_{\mathrm{B}} T q^{2} / 3 \pi \eta_{0} d
$$

Here $\eta_{0}$ is the solvent viscosity at the temperature $T$. However, if $\delta$ is set equal to 0 in eq $2, \Gamma_{\mathrm{q}}$ is underestimated by $0.2-2 \%$. For example, from the data in Figure 3, we obtain $810 \pm 1 \mathrm{~s}^{-1}$, which is about one percent smaller than $\Gamma_{\text {q,calc }}$. Such a small difference in $\Gamma_{\mathrm{q}}$ from $\Gamma_{\mathrm{q} \text {,calc }}$ may be ascribed to either the size distribution or variation in the base line due to the effects described earlier. Since $K_{2} / K_{1}{ }^{2}$

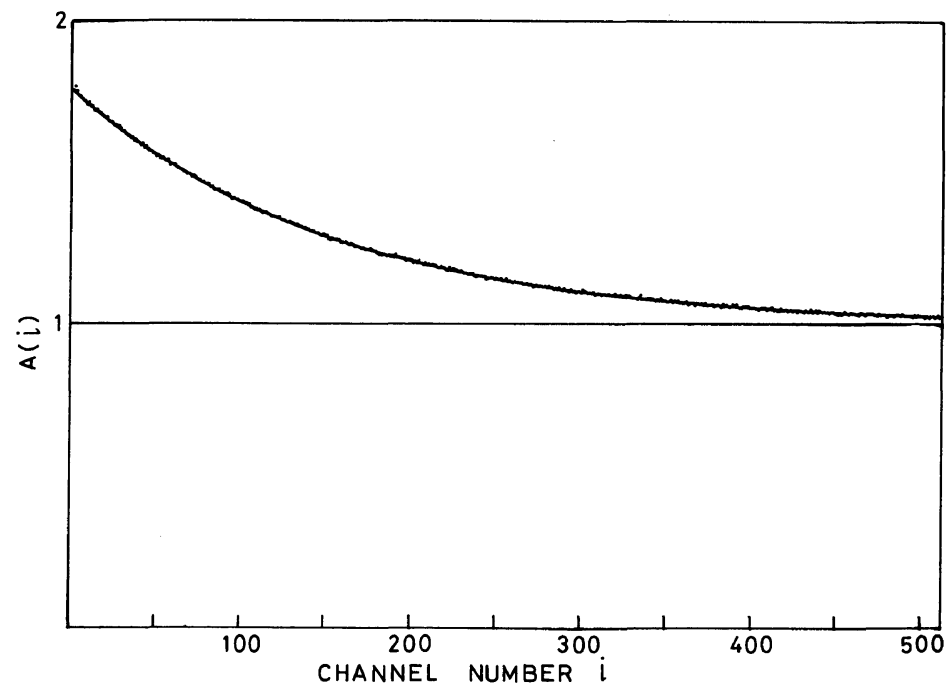

Figure 3. The plot of normalized intensity autocorrelation function $A(i)$ of PS-latex particles $(d=0.176 \mu \mathrm{m})$ in water against the channel number $i . T_{\mathrm{c}}=4 \mu \mathrm{s}, \theta=30^{\circ}, T=25.0^{\circ} \mathrm{C}, \lambda=488 \mathrm{~nm}$, the mean time interval $=150 \mu \mathrm{s}$, and the accumulation number is 2100 . The solid curve represents the curve calculated by the normal eq. fit. 


\section{N. Nemoto, Y. Tsunashima, and M. Kurata}

from the 2 nd order fit is a measure of the width of the size distribution, we calculated this quantity at various scattering angles for the two samples. The values of $K_{2} / K_{1}{ }^{2}$ were found to be less than 0.01 , suggesting that the difference concerned is not due to the particle size distribution. This is consistent with the electron micrographs provided by the distributor, which show the standard deviations of

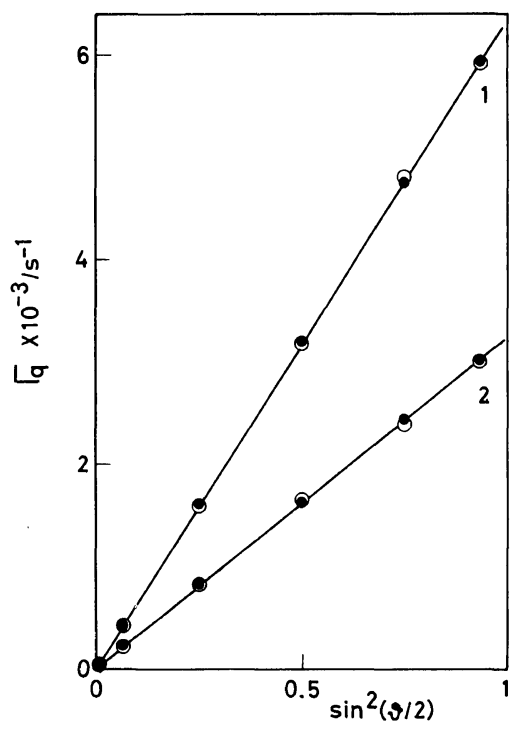

Figure 4. The plot of decay rate $\Gamma_{\mathrm{q}}$ against $\sin ^{2}(\theta / 2)$ for PS-latex particles of two different diameters; 1, $d=0.091 \mu \mathrm{m}$ and $2, d=0.176 \mu \mathrm{m}$. the particle diameter to be $0.0058 \mu \mathrm{m}$ for $d=0.091 \mu \mathrm{m}$ and $0.0023 \mu \mathrm{m}$ for $d=0.176 \mu \mathrm{m}$. This is also consistent with our analysis of the particle size distribution by the histogram method described below. Thus, we tentatively consider that the base line correction is important for an accurate estimation of $\Gamma_{\mathrm{q}}$, and that the preparation of samples and the laser operation must be carried out very carefully.

Since eq 3 should strictly hold for spherically symmetric rigid particles at any scattering angle, $\Gamma_{\mathrm{q}}$ must increase in proportion to $q^{2}$. Figure 4 gives such a plot of $\Gamma_{\mathrm{q}}$ derived by the normal eq. fit against $\sin ^{2}(\theta / 2)$, where the filled circles denote the data obtained by the TI method and the unfilled circles, that by the SR method. Table I lists the experimental values of $\Gamma_{\mathrm{q}}$, those of the translational diffusion coefficient $D\left(=\Gamma_{\mathrm{q}} / q^{2}\right)$ and the theoretical values of $D$. First, it should be noticed that the $\Gamma_{\mathrm{q}}$ values obtained by the two different photon counting schemes agree very well with each other for any scattering angle. Secondly, the plots in Figure 4 are represented by straight lines whose slopes provide the translational diffusion coefficient $D$ of the PSlatices investigated. From these slopes, we evaluated $D$ to be $5.39 \times 10^{-8}$ and $2.76 \times 10^{-8} \mathrm{~cm}^{2} \mathrm{~s}^{-1}$, respectively. Most of these values agree not only with $\Gamma_{\mathrm{q}} / q^{2}$ at all angles, except at $10^{\circ}$, within one percent but also with the theoretical values listed in the last column of Table I within one percent. The deviation at $10^{\circ}$ was about $4-5 \%$, which is larger than $2.5 \%$

Table I. Results of calibration at $T=25^{\circ} \mathrm{C}$ and $\lambda=488 \mathrm{~nm}$

\begin{tabular}{|c|c|c|c|c|c|c|}
\hline \multirow{3}{*}{ Sample } & \multirow{3}{*}{$\frac{\text { Angle }}{\text { degree }}$} & \multicolumn{2}{|c|}{ TI Method } & \multicolumn{2}{|c|}{ SR Method } & \multirow{3}{*}{$\frac{\Gamma_{\mathrm{q} \text {.calc. }} / q^{2} \times 10^{8 \mathrm{c}}}{\mathrm{cm}^{2} \mathrm{~s}^{-1}}$} \\
\hline & & \multirow{2}{*}{$\frac{\Gamma_{\mathrm{q}}}{\mathrm{s}^{-1}}$} & \multirow{2}{*}{$\frac{\left(\Gamma_{\mathrm{q}} / q^{2}\right) \times 10^{8}}{\mathrm{~cm}^{2} \mathrm{~s}^{-1}}$} & \multirow{2}{*}{$\frac{\Gamma_{\mathrm{q}}}{\mathrm{s}^{-1}}$} & \multirow{2}{*}{$\frac{\left(\Gamma_{\mathrm{q}} / q^{2}\right) \times 10^{8}}{\mathrm{~cm}^{2} \mathrm{~s}^{-1}}$} & \\
\hline & & & & & & \\
\hline \multirow{6}{*}{$\begin{array}{l}\text { PS-latex- } \mathrm{H}_{2} \mathrm{O}^{\mathrm{a}} \\
d=0.091 \mu \mathrm{m}\end{array}$} & 10 & 45.8 & 5.12 & 46.0 & 5.15 & \multirow{6}{*}{5.39} \\
\hline & 30 & 427 & 5.41 & 422 & 5.35 & \\
\hline & 60 & 1,610 & 5.46 & 1,590 & 5.41 & \\
\hline & 90 & 3,200 & 5.44 & 3,170 & 5.38 & \\
\hline & 120 & 4,740 & 5.38 & 4,800 & 5.44 & \\
\hline & 150 & 5,930 & 5.40 & 5,910 & 5.39 & \\
\hline \multirow{5}{*}{$\begin{array}{r}\text { PS-latex- } \mathrm{H}_{2} \mathrm{O}^{\mathrm{b}} \\
d=0.176 \mu \mathrm{m}\end{array}$} & 30 & 222 & 2.81 & 217 & 2.75 & \multirow{5}{*}{2.79} \\
\hline & 60 & 817 & 2.78 & 813 & 2.76 & \\
\hline & 90 & 1,620 & 2.75 & 1,640 & 2.79 & \\
\hline & 120 & 2,440 & 2.76 & 2,390 & 2.70 & \\
\hline & 150 & 3,010 & 2.74 & 3,000 & 2.74 & \\
\hline
\end{tabular}

${ }^{\text {a }}$ Concn $=1.04 \times 10^{-3}($ wt $\%)$. ${ }^{\text {b }}$ Concn $=5.5 \times 10^{-4}($ wt $\%)$. ${ }^{\text {c }} \Gamma_{\mathrm{q} . \text { calc. }}=K_{\mathrm{B}} T q^{2} / 3 \pi \eta_{\mathrm{o}} d$. 
as expected from the consideration of the optical alignment. This might be due to the improper positioning of the sample cell which deflected the incident beam toward a smaller scattering angle at the interface between the inner surface of the cell and the water. In fact, such deviation at $10^{\circ}$ was not seen for samples of polystyrene in benzene whose refractive index is much closer to that of the cell glass than is that of water. From these results we thus conclude that the present instrument permits a determination of the translational diffusion coefficients of PS-latex particles within an accuracy of one percent over the range of scattering angles from $30^{\circ}$ to $150^{\circ}$ and also gives a reasonable estimate of $D$ at $10^{\circ}$.

\section{Measurements on Polymer Solutions}

For the test solutions, we chose dilute benzene solutions of a narrow-distribution polystyrene (Toyo Soda, sample code FF-80). A benzene solution of PS with a concentration of $2.146 \times 10^{-3}$ $\mathrm{g} \mathrm{cm}^{-3}$ was prepared and then made optically clean by centrifugation at about $40,000 \mathrm{~g}$ for about $2 \mathrm{~h}$. The concentration of the resulting solution was determined from measurements of the refractive index increment $\Delta n$ by a calibrated differential refractometer. By diluting the solution with dust- free benzene, four solutions with $c=3.92,6.37,8.33$ and $10.81 \times 10^{-4} \mathrm{~g} \mathrm{~cm}^{-3}$ were prepared and used for both static and dynamic light scattering measurements. The results of characterization by a light scattering photometer with a $\mathrm{He}-\mathrm{Ne}$ laser as the light source (Union Giken LS-601) at $T=25^{\circ}$ are given in Table II.

The $A(\tau)$ of the four solutions were measured by the TI method as well as by the SR method at $T=25^{\circ}, \lambda=488 \mathrm{~nm}$ and scattering angles $=30^{\circ}$ and $60^{\circ}$ which satisfy the condition $q\left\langle S^{2}\right\rangle^{1 / 2} \ll 1$ so that eq 3 is applicable for the estimate of $D$ from $\Gamma_{\mathrm{q}}$. Measurements were also made at $10^{\circ}$ for the solution of the highest concentration.

In order to examine the reliability of the DLS instrument with the TI method and to compare the data obtained by the TI and SR methods, another series of benzene solutions of the same polystyrene were prepared and $A(\tau)$ was measured in the range of low polymer concentration from $2.86 \times 10^{-5}$ to

Table II. Characterization of PS in benzene at $25^{\circ}$

\begin{tabular}{cccc}
\hline$M_{w} \times 10^{-5}$ & $M_{w} / M_{n}$ & $\left\langle S^{2}\right\rangle^{1 / 2} \mathrm{~nm} A_{2} \times 10^{4} / \mathrm{mol} \mathrm{cm}^{3} \mathrm{~g}^{-1}$ \\
\hline 7.85 & $1.01^{\mathrm{a}}$ & 39.7 & 3.66 \\
\hline
\end{tabular}

a GPC data from Toyo Soda Co.

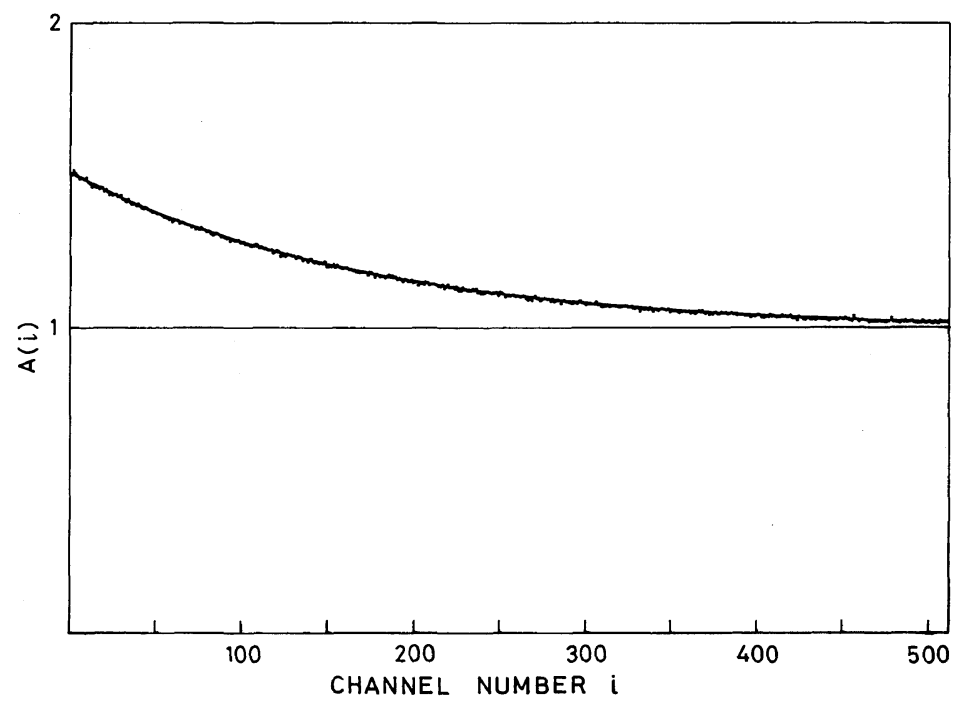

Figure 5. The plot of normalized intensity autocorrelation function $A(i)$ of a dilute benzene solution of the polystyrene sample $\left(M_{w}=785,000\right)$ against the channel number $i$. The concentration of the polymer is $8.33 \times 10^{-4} \mathrm{~g} \mathrm{~cm}^{-3} . T_{\mathrm{c}}=2 \mu \mathrm{s}, \theta=30^{\circ}, T=25^{\circ} \mathrm{C}, \lambda=488 \mathrm{~nm}$, the mean time interval $=29.6 \mu \mathrm{s}$ and the accumulation number is 1000 . The solid curve represents the curve calculated by the normal eq. fit. 


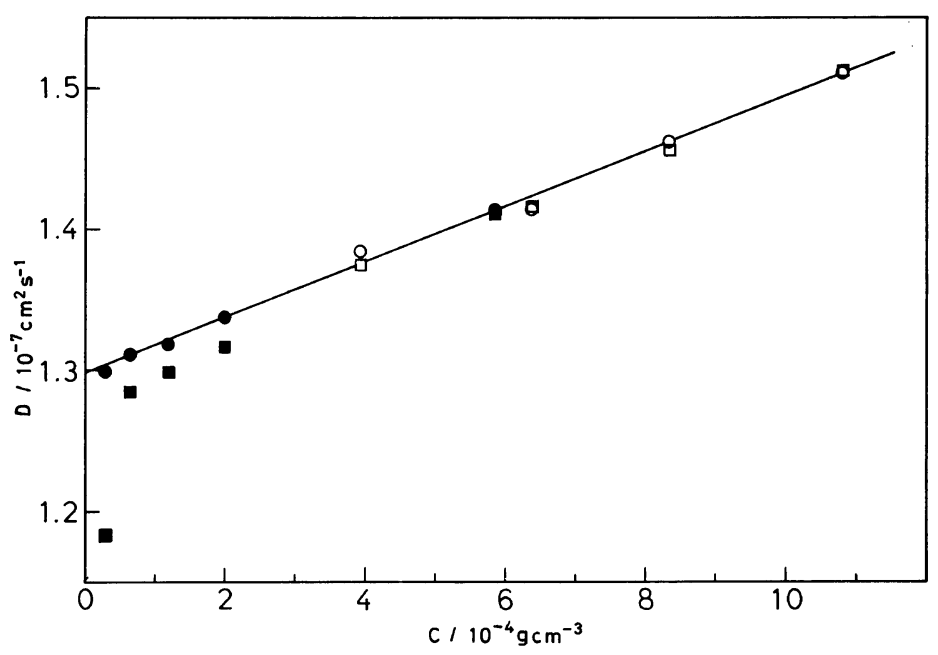

Figure 6. Concentration dependence of the translational diffusion coefficient of the polystyrene sample in benzene at $T=25.0^{\circ} \mathrm{C}$ : $\bigcirc$, average of $D$ obtained at $\theta=30^{\circ}$ and $60^{\circ}$ with the TI method; $\square$, average of $D$ at $\theta=30^{\circ}$ and $60^{\circ}$ with the SR method; $\bigcirc$ at $\theta=30^{\circ}$ with the TI method; $\square, D$ at $30^{\circ}$ with the SR method.

$5.83 \times 10^{-4} \mathrm{~g} \mathrm{~cm}^{-3}$ at a scattering angle of $30^{\circ}$. The comparison was made under same experimental conditions; especially the running time and the maximum delay time were made same for both methods. Figure 5 represents the data for the solution of $c=8.33 \times 10^{-4} \mathrm{~g} \mathrm{~cm}^{-3}$ at $\theta=30^{\circ}$ by the TI method where the clock pulse interval of $2 \mu$ s was employed. The solid curve was calculated from the analysis by the normal eq. fit. $D$ was calculated from $\Gamma_{\mathrm{q}}$ obtained by the normal eq. fit with eq 3 , while the 2nd order fit gave very similar results. The deviations of the base line from unity for measurements at $10^{\circ}$ were $0.6 \%$ at the most and usually less than $0.2 \%$.

In Figure $6, D$ is plotted against the polymer concentration, $c$. For the range of $c$ higher than $2 \times 10^{-4}$, the values of $D$ obtained by the two different photon counting methods agree with each other within an accuracy of one percent, and the dependence of $D$ on $c$ is well represented by a straight line, from which $D_{0}$ and $k_{\mathrm{D}}$ in eq 5 are evaluated as $1.30 \times 10^{-7} \mathrm{~cm}^{2} \mathrm{~s}^{-1}$ and $150 \mathrm{~cm}^{3} \mathrm{~g}^{-1}$, respectively.

$$
D=D_{0}\left(1+k_{\mathrm{D}} c\right)
$$

For a range of $c$ lower than $2 \times 10^{-4} \mathrm{~g} \mathrm{~cm}^{-3}$, the $D$ values obtained by the TI method still fall on the straight line at the lowest concentration $c=2.86 \times$
$10^{-5}$, whereas the $D$ values by the SR method show downward deviations from the straight line and the maximum deviation amounts to about 9 percent at $c=2.86 \times 10^{-5}$. It should be noted that, at $c=2.86 \times 10^{-5}$, the intensity ratio of the solution to pure benzene is 1.27 and the amplitude $\beta$ is as low as 0.04 . These results indicate that the TI method is more effective for very weakly scattering materials than the SR method.

According to various dilute polymer solution theories, ${ }^{9}$ the translational diffusion coefficient of a flexible coil at infinite dilution is related to the rootmean-square radius of gyration $\left\langle S^{2}\right\rangle^{1 / 2}$ by eq 6

$$
D_{0}=Q k_{\mathrm{B}} T /\left\langle S^{2}\right\rangle^{1 / 2} \eta_{0}
$$

The proportionality constant $Q$ has been calculated for various polymer-solvent interaction strengths. At the $\theta$ temperature, the Kirkwood-Riseman theory ${ }^{10}$ gives 0.0799 , Zimm theory ${ }^{11} 0.0784$, Kurata-Yamakawa theory ${ }^{12} \quad 0.0784$, and Benmouna-Akcasu theory ${ }^{13} 0.0798$ for $Q$. In good solvents, Benmouna and Akcasu ${ }^{13}$ predict 0.1069 for $Q$. By substitution of $D_{0}$ and $\left\langle S^{2}\right\rangle^{1 / 2}$ given in Table II into eq 6, $Q$ was evaluated as 0.0755 , which is much smaller than the Benmouna-Akcasu value in good solvents, even if we take into account an estimated error of $3 \%$. It is interesting that 0.0755 is close to the above-quoted theoretical values at the $\theta$ 
temperature in spite of the fact that benzene is a good solvent for PS. More detailed studies over a wide range of molecular weight in both good and poor solvents are in progress.

$k_{\mathrm{D}}$ in eq 5 can be calculated from $A_{2}, M$, the partial specific volume of the polymer $V_{1}$ and the coefficient of concentration dependence of the friction coefficient $k_{\mathrm{f}}$. Since $k_{\mathrm{f}}$ was not measured, we applied the theories of Yamakawa ${ }^{14}$ and Imai ${ }^{15}$ (Y.I. theory), and also the theory of Pyun and Fixman (P.F. theory) ${ }^{16}$ for its estimation. In doing this, the hydrodynamic volume of the polymer was calculated from $D_{0}$. The Y.I. and P.F. theories predicted 161.5 and $78 \mathrm{~cm}^{3} \mathrm{~g}^{-1}$ for $k_{\mathrm{D}}$, respectively. The measured value 150 supported the Y.I. theory for good solvents. A few measurements in poor solvents, ${ }^{17-19}$ however, supported the P.F. theory. Thus, much remains to be investigated both experimentally and theoretically in regard to the $k_{\mathrm{D}}$ of flexible polymers.

\section{Measurement on Aqueous Suspensions of Mixtures of PS-Latex Particles}

In previous sections, we demonstrated that our instrument makes possible accurate determination of translational diffusion coefficients of particles of uniform size and also the particles of a monodisperse polymer. In reality, we frequently encounter cases in which either the size distribution of particles or the molecular weight distribution of synthetic polymers is broad. In certain special cases, it is bimodal. Furthermore, the intramolecular relaxation mode or the gel mode combined with the translational diffusive mode makes the analysis of $A(\tau)$ curves of polymer solutions often difficult and ambiguous. Therefore, we examined the performance of our instrument in dynamic measurements on two mixtures of PS-latex particles $(d=0.176$ and $0.091 \mu \mathrm{m})$ in water which gave known bimodal particle distributions. The weight fractions of one component $(d=0.091 \mu \mathrm{m}$ ) were 0.963 and 0.368 for the mixtures A and B respectively. Measurements were made at $T=25^{\circ}, \lambda=488 \mathrm{~nm}$ and $\theta=60^{\circ}$. The data were analyzed using the histrogram method developed by Chu and coworkers ${ }^{8}$ to obtain the $\Gamma_{\mathrm{q}}$ distribution $H\left(\Gamma_{\mathrm{q}}\right)$. Here $H\left(\Gamma_{\mathrm{q}}\right) d \Gamma_{\mathrm{q}}$ is the fraction of the total integrated intensity of light scattered by the particles which obey $\Gamma_{\mathrm{q}}=D q^{2}$ within the increment $d \Gamma_{\mathrm{q}}$. This method needs no apriori knowledge as to the shape of the distribution. We first input, as a trial, a broad unimodal distribution covering the range of $\Gamma_{\mathrm{q}}$ which should be present in the sample, and then derived $H\left(\Gamma_{\mathrm{q}}\right)$ which most closely approximates the experimental curve on the basis of the non-linear least-squares algorithm by Marquardt. ${ }^{20}$ The results are shown in Fig. 7, where (c) and (d) are $H\left(\Gamma_{\mathrm{q}}\right)$ for the mixtures $\mathrm{A}$ and $\mathrm{B}$, and (a) and (b) are those for their components. Figure 7(c) and (d) clearly show that the mixtures have bimodal distributions of $H\left(\Gamma_{\mathrm{q}}\right)$, which display a peak at the position expected from $\Gamma_{\mathrm{q}}$ of the constituent particles. Furthermore, the ratio of the integrated scattered intensities calculated from the peak areas for the respective components is close to the value roughly estimated from their weight fractions and structure factors. The above results demonstrate again that our instrument is capable of giving autocorrelation functions accurate enough for detecting the bimodal distribution of spherical

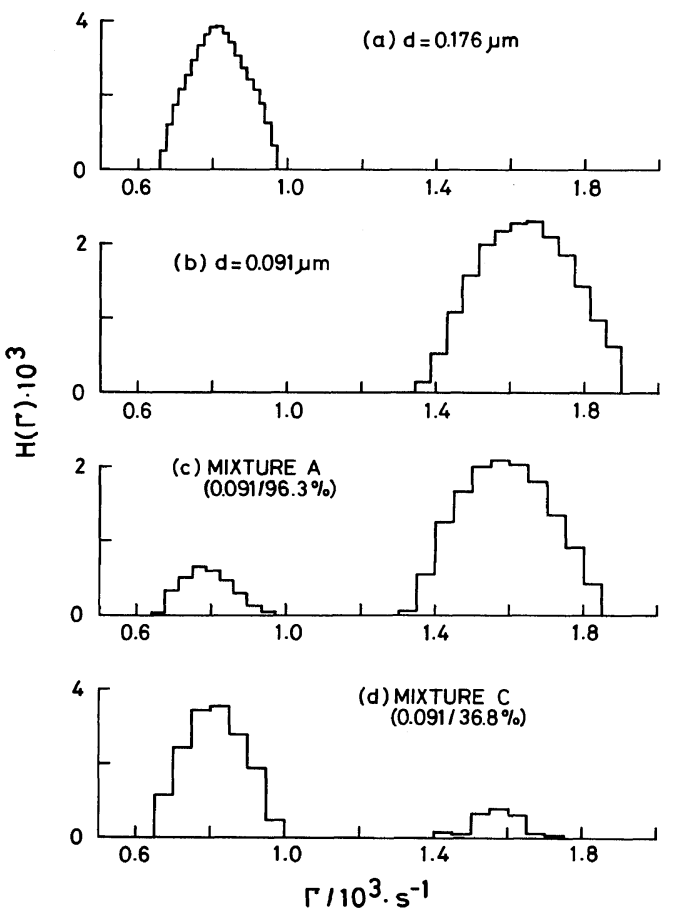

Figure 7. Analysis of uni- and bimodal decay rate distributions by the histogram method. (a) and (b) are results for PS-latex particles considered as almost uniform; (a) $d=0.176 \mu \mathrm{m}$ and (b), $d=0.091 \mu \mathrm{m}$. (c) and (d) are the results for their mixtures; weight fractions of the component with $d=0.091 \mu \mathrm{m}$ are 0.963 and 0.368 , respectively. 
particles.

We also examined dilute solutions of high molecular weight polystyrene to see whether the intramolecular relaxation mode can be extracted from $A(\tau)$ curves overwhelmingly dominated by the translational diffusive mode. We found that the intramolecular relaxation time estimated from the faster decaying component was close to Zimm's first relaxation time at low scattering angles. The details for above will be presented in a subsequent paper. ${ }^{21}$

Acknowledgments. We thank for the cooperation of the Union Giken Co., Osaka for their assistance in constructing our instrument and we also are grateful to Dr. G. Matsumoto of the Electrotechnical Laboratory, Tuskuba for his valuable advice.

\section{REFERENCES}

1. (a) R. Pecora, J. Chem. Phys., 40, 1604 (1964); (b) ibid., 43, 1562 (1965); (c) ibid., 48, 1032 (1968); (d) ibid., 49, 4126 (1968); (e) Ann. Rev. Biophys. Bioeng., 1, 257 (1972)

2. B. J. Berne and R. Pecora, "Dynamic Light Scattering," John Wiley and Sons, Inc., New York, N.Y., 1976.

3. B. Chu, "Laser Light Scattering," Academic Press,
New York, N.Y., 1974.

4. "Photon Correlation Spectroscopy and Velocimetry," H. Z. Cummins and E. R. Pike, Ed., Plenum Press, New York, N.Y., 1976.

5. G. Matsumoto, H. Shimizu, and J. Shimada, Rev. Sci. Instrum., 47, 861 (1976).

6. B. Chu, Pure and Appl. Chem., 49, 941 (1977).

7. D. E. Koppel, J. Chem. Phys., 57, 4814 (1972).

8. E. Gulari, E. Gulari, Y. Tsunashima, and B. Chu, $J$. Chem. Phys., 70, 3965 (1979).

9. H. Yamakawa, "Modern Theory of Polymer Solutions," Harper and Row, Publishers, New York, N.Y., 1971.

10. J. G. Kirkwood and J. Riseman, J. Chem. Phys., 16, 565 (1948)

11. B. H. Zimm, J. Chem. Phys., 24, 269 (1956).

12. M. Kurata and H. Yamakawa, J. Chem. Phys., 29, 311 (1958).

13. M. Benmouna and A. Z. Akcasu, Macromolecules, 11, 1187 (1978)

14. H. Yamakawa, J. Chem. Phys., 36, 2995 (1962).

15. S. Imai, J. Chem. Phys., 50, 2116 (1969).

16. C. W. Pyun and M. Fixman, J. Chem. Phys., 41, 937 (1964).

17. E. Gulari, E. Gulari, Y. Tsunashima and B. Chu, Polymer, 20, 347 (1979).

18. C. C. Han, Polymer, 20, 259 (1979).

19. T. Nose and B. Chu, Macromolecules, 12, 590 (1979).

20. D. W. Marquardt, J. Soc. Ind. Appl. Math., 11, 431 (1963).

21. Polym. J. to be sumbitted. 